\title{
UTILISATION DE SUBSTRATS ARTIFICIELS POUR L'ÉTUDE DE LA FAUNE MACROBENTHIQUE DE LA ZONE LITTORALE ROCHEUSE DES LACS DE MONTAGNE
}

\author{
par H. LAVILLE ${ }^{1}$.
}

Six substrats artificiels immergés au dégel dans la zone rocheuse $(-1-4 \mathrm{~m})$ du lac de Port-Bielh (2 $285 \mathrm{~m})$, relevés à cinq périodes de l'année, sont colonisés par les Chironomides $(90 \%)$ et notamment par les formes libres et mobiles (Tanypodinae, Corynoneura) qui prédominent dans la biocénose littorale.

Ils permettent d'obtenir de précieux renseignements d'ordre qualitatif sur la composition des biocénoses, la microdistribution, les migrations au cours du développement (Psectrocladius sordidellus). Ils s'avèrent convenir plus particulièrement pour l'évaluation quantitative des populations inféodées aux substrats rocheux, permanentes (Limnea) ou temporaires (Paratanytarsus austriacus, Cyrnus cintranus).

Un autre type de substrat doit être envisagé pour les Chironomides constructeurs de fourreaux (Microtendipes, Micropsectra) de même pour les Oligochètes et les Pisidiums, vivant sur ou dans les zones de sédiments accumulés entre les blocs rocheux.

\section{The use of artificial substrata for the study of macrobenthic fauna in the rocky littoral zone of mountain lakes.}

Six artificial substrata immerged during the spring thaw in the rocky zone $(-1$ to $-4 \mathrm{~m})$ of lake Port-Bielh $(2285 \mathrm{~m})$, were sampled five times during the year. They were colonized by chironomids $(90 \%)$ and particularly by free and mobile forms (Tanypodinae, Corynoneura) which predominate in the littoral community. It was possible to obtain valuable information of a qualitative type on the composition of the biocoenosis, the microdistribution, and the migrations during the life cycle (Psectrocladius sordidellus). The artificial substrata were particularly suitable for the quantitative evaluation of permanent (Limnea) or temporary (Paratanytarsus austriacus, Cyrnus cintranus) populations limited to a rocky substratum.

Another type of substratum must be considered for chironomids constructing cases (Microtendipes, Micropsectra) as well as for oligochaetes and Pisidium, all living on or in sediments which accumulate between rocky boulders.

\section{Introduction.}

Les systèmes de prélèvements des substrats vaseux (drague, carottier) sont inutilisables dans les zones littorales rocheuses des

1. Laboratoire d'Hydrobiologie, Université Paul-Sabatier, 118, route de Narbonne, 31077 Toulouse Cedex. France. 
lacs oligotrophes et plus particulièrement dans les lacs d'altitude.

Du fait de ces difficultés d'approche, la faune vivant sur les pierres de la zone littorale a été peu étudiée. Ce son!, 1§ plus souvent, les parties directement accessibles de la zone eulittorale (0 $0,5 \mathrm{~m}$ ) sensu Ruttner (1962) qui ont été prospectées notamment par Ehrenberg (1957) dans les lacs du Holstein oriental et Dusoge (1966) en Pologne.

La zone de prospection peut être étendue grâce à des appareils mis en œuvre par des plongeurs (Rigler 1972, Finnish IBP/PM Group 1969).

Dans les lacs, l'utilisation de substrats artificiels pour l'étude du périphyton est courante. Cette méthode fournit des indications sur la croissance des algues et, dans certaine mesure, permet des observations quantitatives d'un matéricl difficile à récolter sur les substrats naturels.

Pour ce qui concerne le périphyton animal «cette unité écologique indépendante consistant en formes sessiles permanentes mais aussi en formes sessiles temporaires et en formes nageuses》 (Pieczynska 1964), des substrats artificiels ont été proposés par Moon (1935) et Mundie (1956) comme une voie d'accès à ce problème de récolte. De même, leur emploi s'intensifie pour la récolte des organismes macro-invertébrés dans les cours d'eau aux substrats rocheux ou instable (Dickson et al, 1971).

Des substrats artificiels ont été utilisés dans le lac de Port-Bielh $(2285 \mathrm{~m}$ ) afin de tester les modalités de leur colonisation par les invertébrés du benthos et de connaître les possibilités d'utilisation pour une étude quantitative des biocénoses littorales.

\section{Méthodes.}

Le substrat utilisé consiste en une simple cuvette en matière plastique inerte, à fond carré de $31 \mathrm{~cm}$ de côté, haute de $15 \mathrm{~cm}$, d'une superficie de $1 / 10,4 \mathrm{~m}^{2}$. Des pierres granitiques, de dimensions variables, choisies parmi les plus aplaties dans les éboulis côtiers, ont été lavées et agencées de facon à recouvrir entièrement le fond de la cuvette. Le tout est immergé à l'aide de quatre cordes de nylon fixées aux angles de la cuvette et reliées à un flotteur en surface (fig. 1).

Au moment des relevés, réalisés plutôt par temps calme, un filet à vide de maille de $300 \mu$ tendu sur un cadre mélallique lesté glisse le long des cordes et vient s'ajuster sur la partie supérieure de la cuvette. Ce filet empêche le matériel de quitter le substrat au cours de sa remontée qui doit s'effectuer sans à-coups, ce qui devient plus délicat au-delà de $4 \mathrm{~m}$ de profondeur. 6 substrats ont été immergés entre $-1 \mathrm{~m}$ et $-4 \mathrm{~m}$, peu de temps après le dégel 


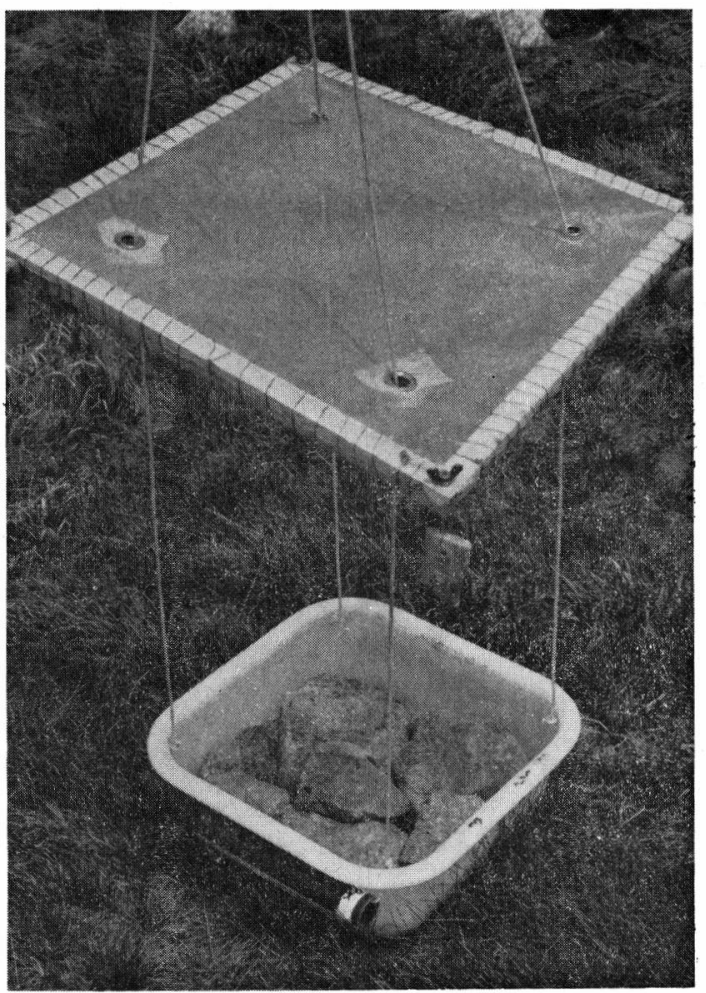

Fig. 1. - Vue du substrat et du filet utilisé lors de son relevé.

du lac, les 12 et 19 juillet 1972, dans une anse sud en pente douce dont le fond est constitué de blocs grantiques à demi-enfouis dans le sédiment. Cette zone représente la zone des Nostocs ou zone infra-littorale sensu Ruttner, récemment définie par Besch et al. (1972). La fréquence et la date de récupération des substrats permettant d'apprécier la durée du temps d'exposition sont récapitulés sur le tableau I.

\begin{tabular}{cccccc}
\hline $\begin{array}{c}\text { Substrat } \\
\mathrm{N}^{\circ}\end{array}$ & $\begin{array}{c}\text { Profondeur } \\
\mathrm{m}\end{array}$ & $\begin{array}{c}\text { Mise en place } \\
1972\end{array}$ & $\begin{array}{c}1^{\mathrm{er}} \text { relevé } \\
1972\end{array}$ & $\begin{array}{c}2^{\text {e }} \text { relevé } \\
1972\end{array}$ & $\begin{array}{c}3^{\mathrm{e}} \text { relevé } \\
1973\end{array}$ \\
\hline & 1 & $12 / \mathrm{VII}$ & $23 / \mathrm{VIII}$ & $7 / \mathrm{XI}$ & $27 / \mathrm{XI}$ \\
2 & 1,5 & $12 / \mathrm{VII}$ & $12 / \mathrm{XX}$ & $7 / \mathrm{XI}$ & $27 / \mathrm{VI}$ \\
3 & 2 & $12 / \mathrm{VII}$ & $12 / \mathrm{IX}$ & - & $27 / \mathrm{VI}$ \\
4 & 2 & $19 / \mathrm{VII}$ & $10 / \mathrm{X}$ & - & $14 / \mathrm{VI}$ \\
5 & 2,5 & $19 / \mathrm{VII}$ & $10 / \mathrm{X}$ & - & $14 / \mathrm{VI}$ \\
6 & 4 & $19 / \mathrm{VII}$ & - & - & $27 / \mathrm{VI}$ \\
\hline
\end{tabular}

Tableau I. - Dates de mise en place et de relevés des substrats. 
5 substrats ont été relevés une ou deux fois entre août et novembre. Tous les six ont été de nouveau émergés au dégel de l'année suivante. Ils donnent des informations sur le mode de colonisation des espèces après un (août), deux (septembre), trois (octobrenovembre), huit ou onze (juin 1973) mois d'exposition.

\section{Résultats.}

La liste et le nombre des espèces récoltés sur chaque substrat à cinq périodes de l'année (Tableau II) permet les observations suivantes :

- Le 23 août, après cinq semaines d'exposition, 7 espèces sont apparues sur le substrat $n^{\circ} 1$ : Limnea, Cloeon, Sialis et 4 Chironomides. Avec 63 individus la colonisation apparaît restreinte et assurée en majeure partie par des larves libres et mobiles ( $A b l a-$ besmyia : $43 \%$; Corynoneura : $36 \%$ ). Notons la présence de larves aux stades III et IV de Paratanytarsus austriacus, formes fixées construisant de longs tubes sinueux faits de particules de sédimentation et d'algues filamenteuses agglomérées, à la surface des pierres : elle témoigne de la mobilité de ces larves à tous les stades du développement et de leur aptitude à construire des fourreaux pendant toute la vie larvaire.

- Après deux mois, deux substrats à -1,5-2 m hébergeaient 15 espèces et une moyenne de 268 individus dont $94 \%$ de Chironomides : $10 \%$ d'entre cux sont de jeunes larves II issues de la nouvelle génération. La vie quasi planctonique des larvules, à la recherche d'un biotope convenable, favorise leur dispersion spatiale et rend possible la colonisation de substrats neufs notamment par les jeunes larves de Microtendipes pedellus, Heterotrissocladius marcidus, Paratanytarsus austriacus. $90 \%$ des Chironomides recueillis sont des larves libres arrivant au terme de leur développement, toujours avec la même prédominance d'Ablabesmyia et de Corynoneura.

Après trois mois d'immersion à $-2-2,5 \mathrm{~m}$ les substrats 4 et 5 récoltaient 17 espèces et 306 individus. Les Chironomides prédominent $(87 \%): 44 \%$ sont des larves II de Psectrocladius sordidellus, forme dominante de la zone littori-profonde vaseuse (7$19 \mathrm{~m})$ et $32 \%$ des larves de Cricotopus pirifer. Ces dernières abondent surtout entre 7 et $15 \mathrm{~m}$ (Laville 1972). Plus récemment nous avons obtenu quelques rares exemplaires dans des pièges à émergence placés en zone littorale. La constance des larves de cette espèce sur les substrats artificiels et sa fréquence $(13,05 \%$ de la totalité des récoltes) semblent indiquer que la densité de $C$. pirifer en zone littorale est supérieure à celle révélée par les émergences.

Limnea peregra $(4,78 \%$ du total des récoltes) est plus abondam- 
(5)

H. LAVILLE

167

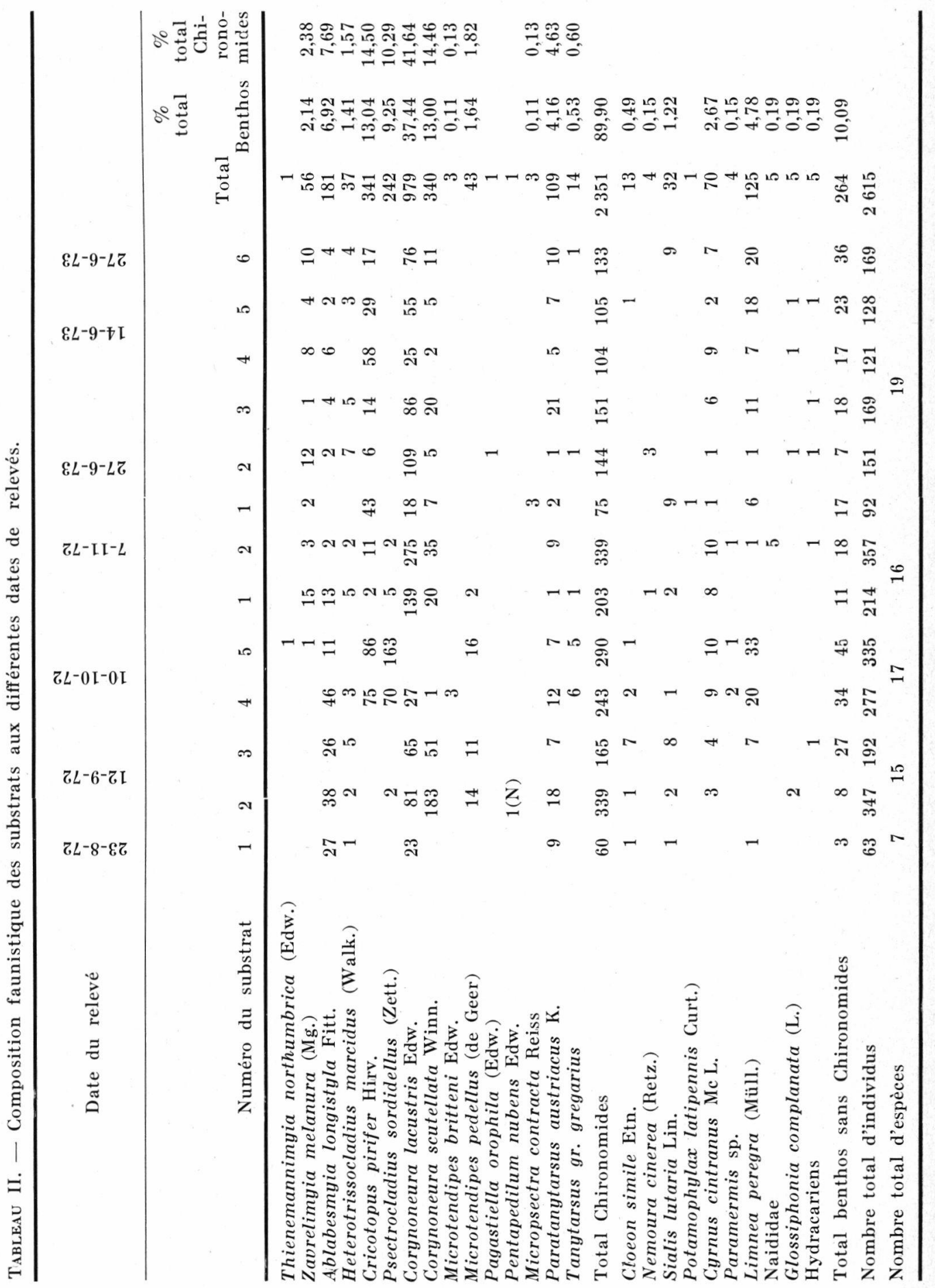


ment représentée sur les substrats artificiels que dans les prélèvements benthiques réalisés par aspiration ou par dragage dans la zone littorale $(0,2 \%$ de la faune benthique : Dupin et Giani 1972). Ces mollusques vivent essentiellement sur la surface des pierres ce qui les rend difficilement accessibles aux appareils de prélèvements manœuvrés depuis la surface. Cinq pontes de Limnées, contenant chacune 5 à 20 embryons, et 19 jeunes Limnées juste écloses ont été récoltées sur les substrats en octobre : elle renseignent sur la reproduction de ce gastéropode.

- Les substrats 1 et 2, replacés respectivement le 23 août et le 12 septembre, ont été relevés, le premier au bout de deux mois et demi, le deuxième après deux mois d'exposition. Pendant ce temps 16 espèces ont colonisé avec en moyenne 285 individus par substrat, essentiellement (235) des Corynoneura : $8 \%$ all stade II, $92 \%$ au stade III.

On note la nette diminution de Psectrocladius sordidellus (3\%) dont les larves ont migré vers la zone littori-profonde où ils dominent entre $11 \mathrm{~m}$ et $14 \mathrm{~m}$ (Laville 1972). De même, la forte régression des Microtendipes, avec seulement deux larves au dernier stade, dénote la désaffection de ces larves détritivores pour les substrats durs au profit de biotopes vaseux.

- Au mois de juin 73 , pendant le dégel (14 juin) ou peu après (27 juin) avant le début des émergences des formes semi-sessiles, les six substrats avaient recueilli 19 espèces. Les Corynoneura y sont toujours dominants. L'absence de Microtendipes de même celle de Psectrocladius confirment leur localisation sur les fonds vaseux, littoraux (Microtendipes) ou profonds (Psectrocladius) convenant mieux à leur mode de vie.

\section{Analyse des résultats.}

Les récoltes d'insectes adultes et d'exuvies nymphales dans les pièges à émergence placés en zone littorale et les prélèvements benthiques réalisés par aspiration sur les rochers ou à l'aide d'une drague Petersen dans les aecumulations de sédiments plus fins (Laville 1972, Dupin et Giani 1972) ont mis en évidence une communauté de la zone rocheuse littorale (Groupement III : $-\mathbf{2}$ à -7 m) du lac de Port-Bielh caractérisée par trois espèces : 2 Chironomides : Microtendipes pedellus et Corynoneura lacustris, un trichoptère : Cyrnus cintranus.

La comparaison de ces résultals avec ceux fournis par les substrats 3 à $6(-2$ à $-4 \mathrm{~m})$ relevés au dégel apparaît dans le tableau III. 8 espèces sont constamment présentes sur les substrats à toutes les dates de relevés, au moins après deux mois d'exposition et, pour ce qui concerne les Chironomides aux trois derniers 
stades de leur développement : Ablabesmyia longistyla, Corynoneura lacustris, C. scutellata, H. marcidus, P. austriacus, Limnea peregra, Cyrnus cintranus, Sialis lutaria. Il s'agit de formes libres, à l'exception de $P$. austriacus et $C$. cintranus : les premières construisent de longs fourreaux refuges sur les pierres, les deuxièmes, tout comme les représentants de la famille des Polycentropodidae, élaborent des filets-pièges en fils de soie sur ou sous les pierres (Edington 1964). Ces 8 espèces font partie du cortège des formes dominantes de la biocénose littorale.

Certaines espèces fréquentes, de cette biocénose, n'ont colonisé les substrats qu'accidentellement (Micropsectra contracta) ou temporairement aux stades jeunes de leur développement (Microtendipes). Signalons que les pièges à émergence utilisés, d'une superficie de $0,25 \mathrm{~m}^{22}$, peuvent couvrir plusieurs microhabilats (rochers et zones d'accumulation de vase et de sable). Ces deux Chironomides détritivores vivent à la surface de la vase où ils construisent soit des fourreaux courts (Microtendipes) soit des tubes beaucoup plus longs que leur corps (Micropsectra) (Walshe 1951). Leur faible densité dans les substrats artificiels paraît liée au fait que ces derniers offraient un biotope exclusivement rocheux.

De même l'absence d'Oligochètes et de Pisidium recensés dans la zone littorale par d'autres modes d'investigation (Dragage Petersen, Pompage) est-elle encore liée à la nature du substrat offert, ces formes, également détritivores, vivant à l'inlerface oxydo-réduite du sédiment (Jonasson 1972). Déjà Moon (1935) constatait l'inefficacité de ses substrats pour récolter Oligochètes et Pisidiums, les gros invertébrés — Plécoptères, Ephéméroptères, Trichoptères, Hirudinées, Gammares et Aselles - colonisant mieux ses plateaux que les Chironomides.

La colonisation permanente de nouveaux substrats est assurée en majeure partie par des formes libres et mobiles, au régime alimentaire varié : consommateurs primaires (Cloeon, Limnea); détritivores (Corynoneura, $H$. marcidus); prédateurs (Tanypodinae, Sialis). La prédominance de ces formes rend compte de l'instabilité du peuplement littoral, de son dynamisme qui augmentent les difficultés de son évaluation quantitative. De ce point de vue, une meilleure approche apparaît au niveau des formes fixées constructrices de fourreaux ( $P$. austriacus, $C$. cintranus) dont la fréquence, voire la densité sont comparables à celles décelées par les émergences (Tableau III).

La population de Cricotopus pirifer colonise essentiellement la zone profonde entre 7 et $15 \mathrm{~m}$ (Laville 1972). Sa fréquence en zone littorale où nous l'avons récoltée dans des pièges placés en 1971 est inférieure à $2 \%$ des émergences. Les larves de cette 


\begin{tabular}{|c|c|c|c|c|c|}
\hline & & \multicolumn{2}{|c|}{ Groupement III (2-7 m) } & \multicolumn{2}{|c|}{ Substrats $3-6(2-4 \mathrm{~m})$} \\
\hline & & $\%$ & N. ind. $/ \mathrm{m}^{2}$ & $\%$ & N. ind./m² \\
\hline Z. melanura & . . & 2,76 & 54 & 3,88 & 52 \\
\hline A. longistyla & & 9,44 & 185 & 3,06 & 41 \\
\hline Procladius sp. II & & 2,76 & 54 & - & - \\
\hline H. marcidus & & 5,41 & 106 & 2,32 & 31 \\
\hline C. pirifer & & - & - & 23,30 & 312 \\
\hline C. lacustris & & 29,18 & 572 & 47,34 & 634 \\
\hline C. scutellata & & 7,45 & 146 & 6,95 & 93 \\
\hline$M$. britteni & & 2,29 & 45 & - & - \\
\hline M. pedellus & $\therefore$ & 10,66 & 209 & - & - \\
\hline$M$. contracta & & 5,61 & 110 & - & - \\
\hline P. austriacus & & 7,45 & 146 & 8,51 & 114 \\
\hline C. cintranus & & 4,69 & 92 & 4,63 & 62 \\
\hline Divers $(<2 \%)$ & & 12,29 & 241 & - & - \\
\hline Total & & 99,99 & 1960 & 99,99 & 1339 \\
\hline
\end{tabular}

TABlead IIl. - Densité moyenne et fréquence des formes semi-sessiles récoltées au dégel sur les substrats comparées à celles obtenues en 1971 par piégeages dans la zone $2-7 \mathrm{~m}$ et constituant le groupement III de la zone littorale (Dupin et Giani 1972).

espèce sont bien représentées sur la totalité des substrats artificiels où elles constituent en moyenne $14,5 \%$ des Chironomides récoltés. Ces différences sont peut-être liées au comportement des larves avant la nymphose. En Camargue, J.-N. Tourenq (comm. verb.) a constaté que les larves du dernier stade de certains Cricotopus nagent vers la surface à la recherche d'un support pour se nymphoser. De plus, leur phototropisme positif les conduirait à éviter davantage les pièges de faible profondeur que ceux immergés vers 7 - $8 \mathrm{~m}$ dans la zone profonde, moins bien éclairée.

\section{VITESSE DE COLONISATION DES SUBSTRATS.}

Au lac de Windermere, quatre semaines d'exposition suffisent pour obtenir sur les substrats une faune comparable à celle des milieux voisins (Moon 1935). Le temps plus long (environ deux mois) nécessaire pour une colonisation convenable des substrats au lac de Port-Bielh peut être attribué au fait que les pierres des substrats sont initialement dépourvues de couverture algale nutritionnelle; l'utilisation de pierres prélevées dans le lac, à l'exemple de Moon, était ici plus délicate du fait de la prédominance de petites larves de Chironomides (Corynoneura) difficiles à séparer du substrat.

Par suite, l'allongement du temps de colonisation par les macroinvertébrés benthiques peut correspondre au temps nécessaire pour 
une colonisation convenable par les algues épilithiques. Capblaneq (1973) a montré que le taux de croissance des algues sur les substrats vierges était très faible dans la zone littorale du lac de Port-Biclh.

\section{Conclusions.}

Les formes libres et mobiles - Tanypodinae (Ablabesmyia, Zavrelimyia), Corynoneura, H. marcidus, Limnea, Sialis, Cloeon sont les premières à coloniser les nouveaux substrats.

Les variations de densité dans les divers substrats sont en partic liées à leur mobilité. Plus stables sont les populations de formes fixées ( $P$. austriacus, $C$. cintranus) dont la fréquence est très comparable à celle obtenue à partir des pièges à émergence.

Les différences de peuplement des substrats concernent essentiellement des formes secondaires ou rares de la biocénose. L'absence de quelques formes, abondantes dans les pièges, peut s'interpréter comme résultant de la nature du substrat utilisé : il s'agit pour la plupart de formes inféodées aux zones de vase accumulée entre les rochers.

Compte tenu de la diversité spécifique de la zone littorale, des nombreux microhabitats et de la prédominance des formes mobiles, on ne peut espérer des résultats quantitatifs valables qu'à condition de multiplier le nombre et de diversifier la nature de ces substrats pour qu'ils soient représentatifs de tous les habitats naturels existant dans le lac.

Utilisés conjointement à d'autres méthodes de récolte, ces substrats peuvent fournir de précieux renseignements tant d'ordre qualitatif (composition des biocénoses, étude de la croissance des larves, micro-distribution, migration au cours du cycle de développement) que quantitatifs, notamment pour la faune inféodée aux substrats rocheux, permanente (Limnea) ou temporaire ( $P$. austriacus, C. cintranus).

\section{TRAVAUX GITÉS}

Besch (W. K.), Backhaus (D.), Capblance (J.) et Lavandier (P.) 1972. - Données écologiques sur les algues benthiques de haute montagne dans les Pyrénées. I. Diatomées. Annls Limnol., 8 (2) : 103118.

CaPblance (J.). 1973. - Phytobenthos et productivité primaire d'un lac de haute montagne dans les Pyrénées centrales. Annls Limnol., 9 (3) : 193-230.

Dickson (K. L.), Cairns (J.) Jr and Arnold (J.C.). - An evaluation of the use of a basket artificial substrate for sampling macroinvertebrate organisms. Trans. Amer. Fish. Soc., 100 (3) : 553-559. 
Dupin (B.) et Giani (N.). 1972. — Étude des populations benthiques d'un lac de haute montagne, le lac de Port-Bielh $(2285 \mathrm{~m})$, Hautes-Pyrénées .Thèse $3^{\circ}$ cycle : U.P.S. Toulouse, $(28$ juin 1972).

Dusoge (K.). 1966. - Composition and interrelations between macrofauna living on stones in the littoral of Mikolajskie lake. Ekol. Pol., Sér. A, XIV (39) : 755-762.

Edington (J. M.). 1964. - The taxonomy of the British Polycentropid larvae (Trichoptera). Proc. Zool. Soc. Lond., 143 (2) : 281-300.

Ehrenberg (H.). 1957. - Die Steinfauna der Brandungsufer ostholsteinischer Seen. Arch. Hydrobiol., 53 : 87-159.

FINNISH IBP-PM Group. 1969. - Quantitative sampling equipement for the littoral benthos. Int. Rev. Ges. Hydrobiol., 54 (2) : 185-193.

Jonisson (P. M.). 1972. - Ecology and production of the profundal benthos in relation to phytoplancton in lake Esrom. Ölkos, suppl. $14: 1-148$.

LAville (H.). 1972. - Recherches sur les Chironomides (Diptera) lacustres du massif de Néouvielle (Hautes-Pyrénées), deuxième partie : Communautés et Production. Annls Limnol., 7 (3), 1971 : 335-414.

Moon (H. P.). 1935. - Methods and apparatus suitable for the investigation of the stony littoral of oligotrophic lakes. Int. Rev. Ges. Hydrobiol., 32 : 319-333.

Mundie (J. H.). 1956. - A bottom sampler for inclined rock surface in lakes. J. Anim. Ecol., 25 : 429-432.

Pieczy s ska (E.). 1964. - Investigations on colonisation of new substrates by Nematodes (Nematoda) and other periphyton organisms. Ekol. Pol., Sér. A, XII : 185-214.

Rigler (F. H.). 1972. - The Char lake project. A study of energy flow in a high arctic lake. Productivity problems of freshwaters Warszawa-Krakow. Edit. Kajak (Z.) \& Hillbricht-Ilkowska (A.) : 287-300.

Ruttner (F.). 1962. - Grundriss der Limnologie. V. Gruyters et Co., Berlin, $3^{\circ}$ Ed., $339 \mathrm{p}$.

Walshe (B. M.). 1951. - The feeding habits of certain Chironomid larvae (subfamily Tendipedinae). Proc. Zool. Soc. Lond., 121: 63-79. 